\title{
Single-Crystalline InGaAs Nanowires for Room-Temperature High-Performance Near-Infrared Photodetectors
}

\author{
Huang Tan ${ }^{1}$ Chao Fan $^{1} \cdot$ Liang Ma $^{1} \cdot$ Xuehong Zhang ${ }^{1} \cdot$ Peng Fan ${ }^{1} \cdot$ Yankun Yang ${ }^{1}$. \\ Wei Hu${ }^{1} \cdot$ Hong Zhou ${ }^{1} \cdot$ Xiujuan Zhuang ${ }^{1} \cdot$ Xiaoli Zhu ${ }^{1} \cdot$ Anlian Pan ${ }^{1}$
}

Received: 22 May 2015/Accepted: 20 July 2015/Published online: 21 September 2015

(C) The Author(s) 2015. This article is published with open access at Springerlink.com

\begin{abstract}
InGaAs is an important bandgap-variable ternary semiconductor which has wide applications in electronics and optoelectronics. In this work, single-crystal InGaAs nanowires were synthesized by a chemical vapor deposition method. Photoluminescence measurements indicate the InGaAs nanowires have strong light emission in near-infrared region. For the first time, photodetector based on as-grown InGaAs nanowires was also constructed. It shows good light response over a broad spectral range in infrared region with responsivity of $6.5 \times 10^{3} \mathrm{~A} \mathrm{~W}^{-1}$ and external quantum efficiency of $5.04 \times 10^{5} \%$. This photodetector may have potential applications in integrated optoelectronic devices and systems.
\end{abstract}

Keywords InGaAs $\cdot$ Nanowire $\cdot$ Near-infrared $\cdot$ Photodetector

\section{Introduction}

One-dimensional semiconductor nanowires have attracted considerable attention as unique building blocks for various interesting applications in integrated electronic and optoelectronic devices and systems, such as nanoscale lasers, fieldeffect transistors, solar cells, and photodetectors [1-8]. Among them, nanowire-based photodetectors have aroused wide interests as potential key functional units in on-chip information communication and processing $[9,10]$. Although

Huang Tan and Chao Fan contributed equally to this work.

Electronic supplementary material The online version of this article (doi:10.1007/s40820-015-0058-0) contains supplementary material, which is available to authorized users.

Xiaoli Zhu

zhuxiaoli@hnu.edu.cn

Anlian Pan

anlian.pan@hnu.edu.cn

1 Key Laboratory for Micro-Nano Physics and Technology of Hunan Province, School of Physics and Electronics, and State Key Laboratory of Chemo/Biosensing and Chemometrics, Hunan University, Changsha 410082,

People's Republic of China much work has been devoted to investigating nanowire-based photodetectors in the past few years [10-16], most of the reported nanowire detectors are limited in the visible and ultraviolet spectral regions $[9,11,13,14]$, and little work is conducted in the infrared region $[15,16]$. III-V semiconductor nanowires with narrow bandgap are considered as promising candidates for constructing infrared photodetectors. To date, infrared photodetectors based on InAs, InPAs, and InGaSb nanowires have been reported $[8,10,16,17]$.

As an important III-V ternary semiconductor, InGaAs are expected to have potential bandgap tunability from the nearinfrared (NIR) to mid-infrared (MIR) region $\left(0.35 \leq E_{\mathrm{g}}\right.$ $\leq 1.42 \mathrm{eV}$ ). Due to its tunable bandgap, as well as high electron mobility and small leakage current, $\mathrm{In}_{\mathrm{x}} \mathrm{Ga}_{1-\mathrm{x}}$ As nanowires have been widely used in optoelectronic devices, such as NIR emission lasers, photovoltaics, and field-effect transistors [18-20]. Till now photodetectors based on InGaAs quantm dots and films have been fabricated. However, the performances of these devices are relatively poor. It is desirable to fabricate $\mathrm{InGaAs}$ photodetectors with high performance based on other nanomaterials. To the best of our knowledge, there is no report on roomtemperature infrared photodetector based on InGaAs nanowires.

In this work, InGaAs nanowires were first synthesized via a simple chemical vapor deposition (CVD) method. Raman and photoluminescence measurements illustrate 
that the as-grown nanowires are high-quality single crystals. More importantly, integrated infrared photodetectors were constructed by using these as-grown nanostructures. The achieved devices exhibit good photoresponse in a broad spectral range from 1100 to $2000 \mathrm{~nm}$, in which the responsivity (R) and the external quantum efficiency (EQE) are comparable with those infrared photodetectors based on other III-V nanostructures at room temperature $[8,16]$. Our results may possess important applications in integrated photonics and optoelectronics devices.

\section{Experimental}

CVD method was used to grow InGaAs nanowires. Briefly, InAs power (Alfa Aesar, $99.99 \%$ ) and GaAs power (Alfa Aesar, $99.99 \%$ ) were mixed equivalently and placed into the heating zone of the furnace. Silicon wafers coated with 5-nmthick gold film were placed downstream with a distance of $24 \mathrm{~cm}$ away to the center of tube furnace to collect the deposited InGaAs nanowires. Before heating Ar mixed with $10 \% \mathrm{H}_{2}$ gas flowed through the horizontal quartz tube at a rate of $45 \mathrm{sccm}$ and the pressure maintained at 3 Torr. The temperature of the powder sources was set to $860{ }^{\circ} \mathrm{C}$ and that of the substrate was approximately $520{ }^{\circ} \mathrm{C}$ (schematic diagram of the experimental setup and the temperature gradient in the furnace see Fig. S1). After 90 min of growth at $860^{\circ} \mathrm{C}$, the temperature was reduced naturally to room temperature.

The morphology of the as-prepared sample was characterized by field emission scanning electron microscopy (FESEM, Hitachi S-4800). The phase characterization was identified using XRD (Rigaku D/Max 2500). Transmission electron microscopy (TEM, Tecai F20) combined with energy-dispersive X-ray spectroscopy (EDX) was used to investigate the microstructure and elemental composition. Raman spectrum was performed by a $\mu$-Raman (WITec alpha-300) system excited with a $488 \mathrm{~nm}$ argon ion laser. The photoluminescence (PL) measurements were carried out on a home-built IR micro-PL setup. The samples were excited by a passively mode-locked Ti:sapphire laser. (Spectra Physics Tsunami, $800 \mathrm{~nm}, 150 \mathrm{fs}$ pulse duration, $80 \mathrm{MHz}$ repetition rate.) The PL signal was collected and detected using a spectrometer (HORIBA iHR 550) equipped with a liquid nitrogen-cooled InGaAs photodiode detector $(1300-2300 \mathrm{~nm})$. The current-voltage $(I-V)$ characteristics of the photodetectors were measured using a Keithley 4200 .

\section{Results and Discussion}

The typical SEM image of the as-grown sample is shown in Fig. 1a. The nanowires with uniform diameter and tens of micrometers length were deposited in high yields on the $\mathrm{Si}$
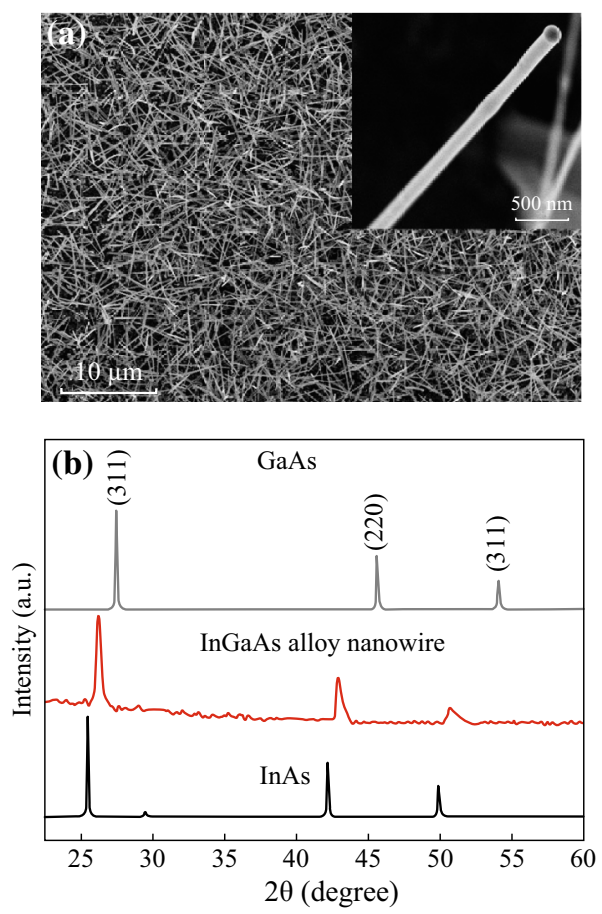

Fig. 1 a Typical SEM image of the morphology of the as-grown nanowires. $\mathbf{b}$ XRD patterns of the as-deposited alloy nanowires

substrate. The inset of Fig. 1a shows a high-magnification SEM image of a typical nanowires with a diameter of about $150 \mathrm{~nm}$. Figure $1 \mathrm{~b}$ represents the XRD pattern of the asdeposited alloy nanowires. For contrasting study, XRD patterns of pure InAs (bottom, JCPDS No. 88-2489) and GaAs (top, JCPDS No. 89-3314) are also plotted. All the diffraction peaks located between those of zinc blende InAs (bottom, JCPDS No. 88-2489) and GaAs (top, JCPDS No. 89-3314) single crystals are clearly seen, confirming the as-grown sample is an InGaAs alloy with zinc blende crystallographic phase. There are no characteristic peaks from other oxides or crystalline impurities, suggesting the formation of high-purity InGaAs nanowires.

Figure 2a shows the TEM image of a representative nanowires. It can be seen that the surface of the nanowire is smooth, and there is a typical spherical catalytic particle at the tip of the nanowire evidenced by the typical metalcatalyzed vapor-liquid-solid (VLS) growth mechanism. Figure $2 \mathrm{~b}$ displays the high-resolution TEM (HRTEM) image taken from the nanowire (the pink rectangle in Fig. 2a). It demonstrates that the nanowire has singlecrystalline zinc blende (ZB) structure. The plane spacing is 0.341 and $0.286 \mathrm{~nm}$, which is corresponding well to (111) and (200) lattice planes in the In-rich thin-film counterparts [21], respectively. Figure 2c illustrates the corresponding EDX spectra of the nanowire. The peaks of In, Ga, and As have strong intensities and their atomic ratio is close to 0.65:0.35:1, demonstrating that the composition of the achieved wires is $\mathrm{In}_{0.65} \mathrm{Ga}_{0.35}$ As alloys (the detected $\mathrm{Cu}$ 

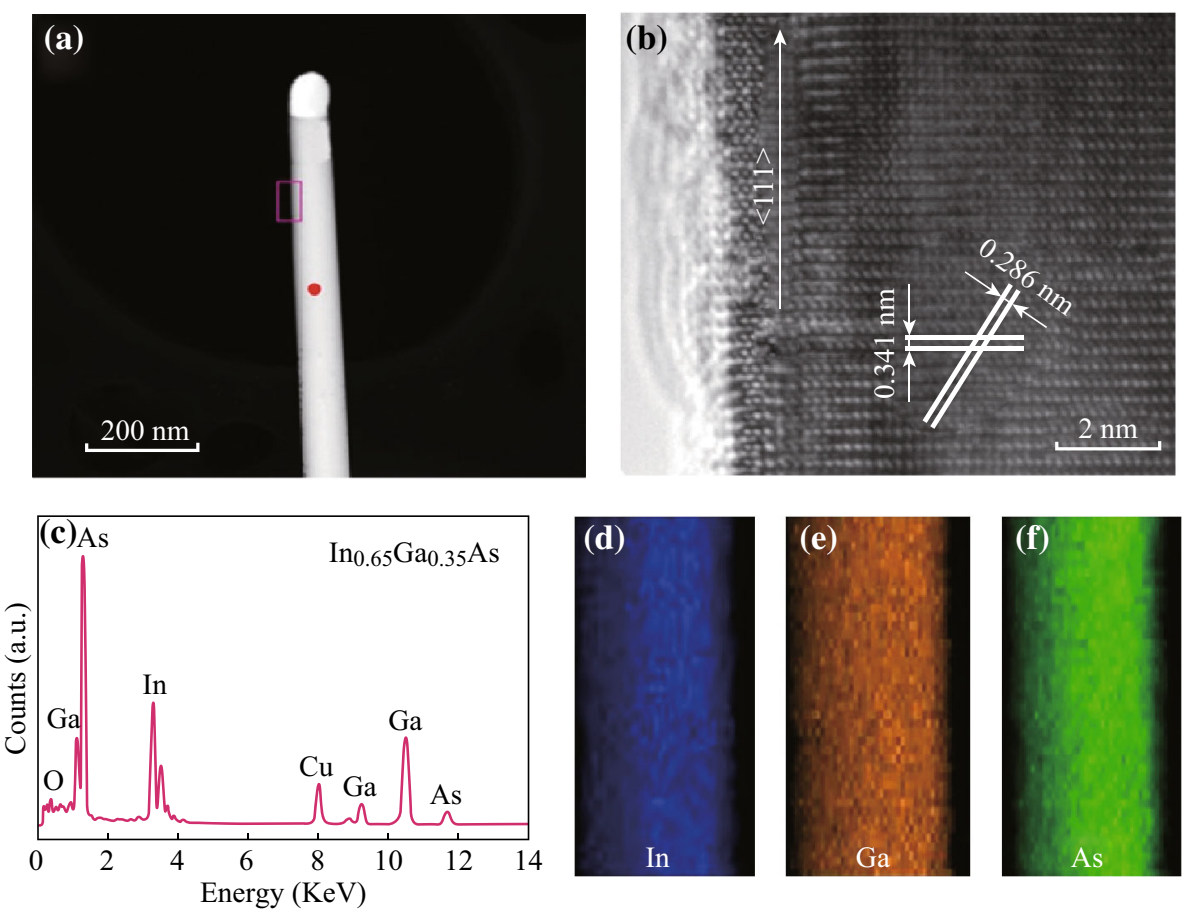

Fig. 2 a TEM image of a representative single InGaAs nanowire. b HRTEM image taken from the pink rectangle of Fig. 2a. c Corresponding EDX profiles measured from the red position of the nanowire. d-f $2 \mathrm{D}$ elemental mapping for the three detected elements: In, Ga, and As, respectively

element originates from the copper grid). Figure $2 \mathrm{~d}-\mathrm{f}$ show the two-dimensional (2D) elemental mapping of this nanowire. As can be seen, In, Ga, and As are homogeneously distributed across the whole nanowire.

In order to further characterize the microstructural properties of the as-grown $\mathrm{In}_{0.65} \mathrm{Ga}_{0.35}$ As nanowires, we have performed the Raman spectrum measurements. A typical Raman spectrum of the $\mathrm{In}_{0.65} \mathrm{Ga}_{0.35}$ As nanowires is displayed in Fig. 3. Two phonon modes at 223 and $249 \mathrm{~cm}^{-1}$ are clearly seen, which is assigned to the InAs-like and GaAs-like transverse optical phonon modes, respectively. Regarding the peaks position of the two phonon modes, it can be found that

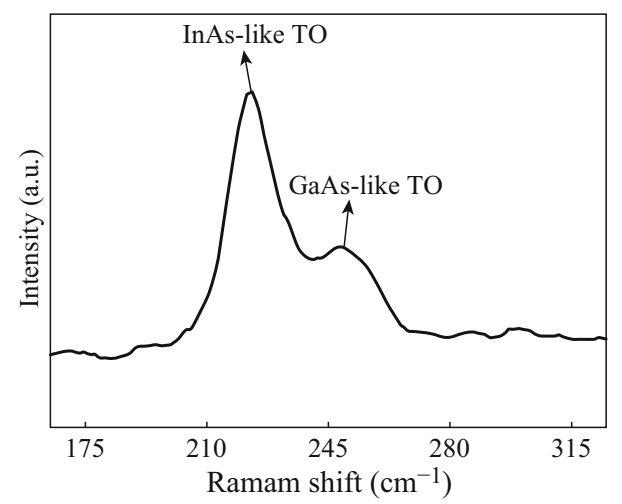

Fig. 3 Raman spectrum of the $\operatorname{In}_{0.65} \mathrm{Ga}_{0.35}$ As nanowires excited with a $488 \mathrm{~nm}$ argon ion laser the experimental data are in agreement well with literature values $[22,23]$. This confirms that the achieved $\operatorname{In}_{0.65} \mathrm{Ga}_{0.35} \mathrm{As}$ nanowires have good compositional homogeneity and highquality crystallization without the stacking disorder.

Figure 4a exhibits the temperature-dependent PL of the $\mathrm{In}_{0.65} \mathrm{Ga}_{0.35} \mathrm{As}$ nanowires excited with an $800-\mathrm{nm}$ femtosecond laser at the temperature from 77 to $270 \mathrm{~K}$. A strong PL emission band with the peak wavelength at $1794 \mathrm{~nm}$ can be observed at $77 \mathrm{~K}$, which is well consistent with the band gap value of In-rich InGaAs alloy nanowires $(0.69 \mathrm{eV})$ grown by MBE (Molecular Beam Epitaxy) at this temperature [24]. The temperature dependence of the band gap is a continuous function with the Varshni's empirical relation $\left(E_{\mathrm{g}}(T)=E_{\mathrm{g}}(0 \mathrm{~K})-\alpha T^{2} /(T+\beta)\right)$ [25]. According to this equation, the fitted $\alpha$ and $\beta$ are $3 \times 10^{-4} \mathrm{eV} \mathrm{K}^{-1}$ and $105 \mathrm{~K}$ for the $\operatorname{In}_{0.65} \mathrm{Ga}_{0.35} \mathrm{As}$ (the calculated process sees Supporting Information). The measured experiment results obtained from PL peak position are consistent with the calculated band gap values as shown in Fig. 4b, which demonstrates that the observed PL is mainly from the bandedge emission of the $\operatorname{In}_{0.65} \mathrm{Ga}_{0.35}$. As nanowires without any observed defect-related emission bands. All of these results are in agreement well with the structural and composition investigations described above, further demonstrating the high-quality of the $\operatorname{In}_{0.65} \mathrm{Ga}_{0.35}$ As nanowires.

To shed light on studying the photoconductance properties of as-grown nanowires, photodetectors were 

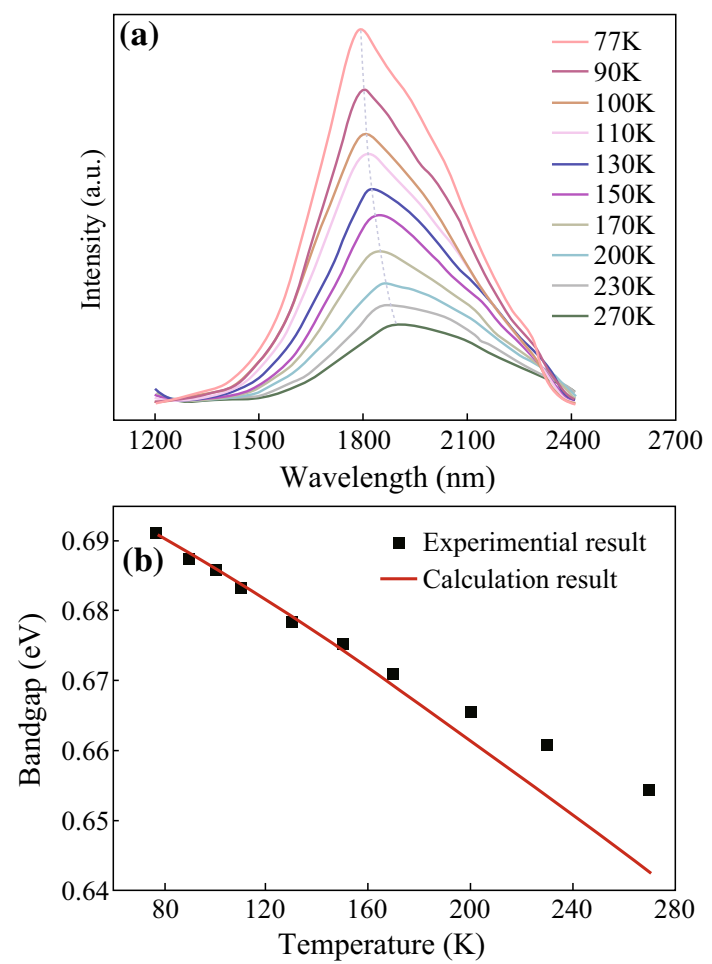

Fig. 4 a Temperature-dependent PL spectra of the $\mathrm{In}_{0.65} \mathrm{Ga}_{0.35} \mathrm{As}$ nanowires under the same laser excitation, the dashed line shows the shift of the PL peak energy with changing the temperature. b Temperature-dependent bandgap of the achieved $\operatorname{In}_{0.65} \mathrm{Ga}_{0.35} \mathrm{As}$ nanowires

constructed using these high-quality $\operatorname{In}_{0.65} \mathrm{Ga}_{0.35}$ As alloy nanowires. The wires were first dispersed on a p-type $\mathrm{Si}$ substrate with 300-nm thickness of $\mathrm{SiO}_{2}$ layer. Photolithography was employed to define the source and drain pattern. $\mathrm{Cr} / \mathrm{Au}(10 / 60 \mathrm{~nm})$ source/drain (S/D) electrodes were made by metal evaporation and lift-off processes. A single InGaAs nanowire connected with the $\mathrm{Cr} / \mathrm{Au}$ Schottky contact electrodes constitutes a typical $\mathrm{M}-\mathrm{S}-\mathrm{M}$ photodetector. Figure 5a shows the $I-V$ curves of a representative nanowire photodetector (see the inset of the figure) with a wire diameter of $d \sim 150 \mathrm{~nm}$ and a channel length of $L \sim 10 \mu \mathrm{m}$, measured in the dark condition and under the illumination of a beam of a monochromatic light with the wavelength of $1100,1300,1500,1600,1800$, and $2000 \mathrm{~nm}$ (light intensity $P_{\text {in }}=15.8 \mathrm{~mW} \mathrm{~cm}^{-2}$ ) at room temperature, respectively. At a fixed bias voltage of $0.5 \mathrm{~V}$, it can be seen that the dark current is about $144 \mathrm{nA}$, and the largest photo-excited current reaching $\sim 2.5 \mu \mathrm{A}$ was recorded at the wavelength of $1600 \mathrm{~nm}$. The curves show the electric conductance of the devices drastically increases under the light illumination of all the given wavelengths, suggesting good photoresponse ability of the device in near-infrared spectrum.

In order to get the intuitional result of the photoresponse, the calculated spectral responsivity $\left(R_{\lambda}\right)$ and external quantum efficiency (EQE) results of the $\mathrm{In}_{0.65}$ $\mathrm{Ga}_{0.35}$ As nanowire device for different wavelengths ranging from 1000 to $2000 \mathrm{~nm}$ are depicted in Fig. 5b. One can notice that the photoresponse increases gradually as the incident light wavelength changes from 1100 to $1600 \mathrm{~nm}$ and then decreases rapidly with the increase of light wavelength larger than $1600 \mathrm{~nm}$. This indicates that the response spectrum is related to the energy band structure of $\mathrm{In}_{0.65} \mathrm{Ga}_{0.35} \mathrm{As}$ nanowires. The photons with larger energy than band gap are easily capable to excite the electron-hole pairs and therefore enhance the photoconductive sensitivity. These results demonstrate that the device based on $\mathrm{In}_{0.65} \mathrm{Ga}_{0.35} \mathrm{As}$ nanowires has good wavelength selectivity and high photosensitivity to infrared light in the NIR region from 1100 to $2000 \mathrm{~nm}$.

$\mathrm{R}$ and EQE, two key parameters of a photodetector sensitivity to the incident light, can be expressed as $[9,13]$

$R=\frac{I_{\mathrm{ph}}}{P S}$

$E Q E=R \times \frac{h c}{e \lambda}$,

where $I_{\mathrm{ph}}$ is the photocurrent, $P$ is the light intensity, $S$ is the effective illuminated area, $h$ is Plank's constant, $c$ is the velocity of light, and $\lambda$ is the incident light wavelength. The values of $\mathrm{R}$ and EQE at $1600 \mathrm{~nm}$ were calculated to be $6.5 \times 10^{3} \mathrm{~A} \mathrm{~W}^{-1}$ and $5.04 \times 10^{5} \%$ at a bias voltage of $0.5 \mathrm{~V}$, respectively. They are higher than those of conventional infrared photodetectors constructed with InGaAs quantum dots and thin films [26, 27].

It is well known that one-dimensional semiconducting nanowires own higher surface-to-volume ratio and can easily induce trap states at the nanowire surface. Those trap states will significantly affect the transport and photoconduction properties of the nanowire-based photodetectors. It was reported that $\operatorname{In}_{0.65} \mathrm{Ga}_{0.35}$ As nanowire has higher density of surface electrons. Oxygen molecules can be easily adsorbed and combined with free electrons on the nanowire surface to form a low-conductivity depletion layer and result in increase of carrier densities [24]. Under NIR light illumination, the generated electron-hole pairs diffuse into the depletion region and then drift to the surface under the electric field in a radial direction and neutralize the adsorbed oxygen ions. This process releases back the captured free electrons and increases the free electron concentration. It was reported that the photoconductive gain can be expressed as $G=\tau / T_{\mathrm{r}}$, where $\tau$ is the carrier lifetime and $T_{\mathrm{r}}$ is the transition time between electrodes. Because of the existence of an internal electric field, the recombination of carriers will be slowed down and the life time of the photocarrier $(\tau)$ will be prolonged [13]. For $\mathrm{In}_{0.65} \mathrm{Ga}_{0.35}$ As nanowires with a proper radius of $150 \mathrm{~nm}$, the active area of the carriers will be confined by one-dimensional structure and therefore the 

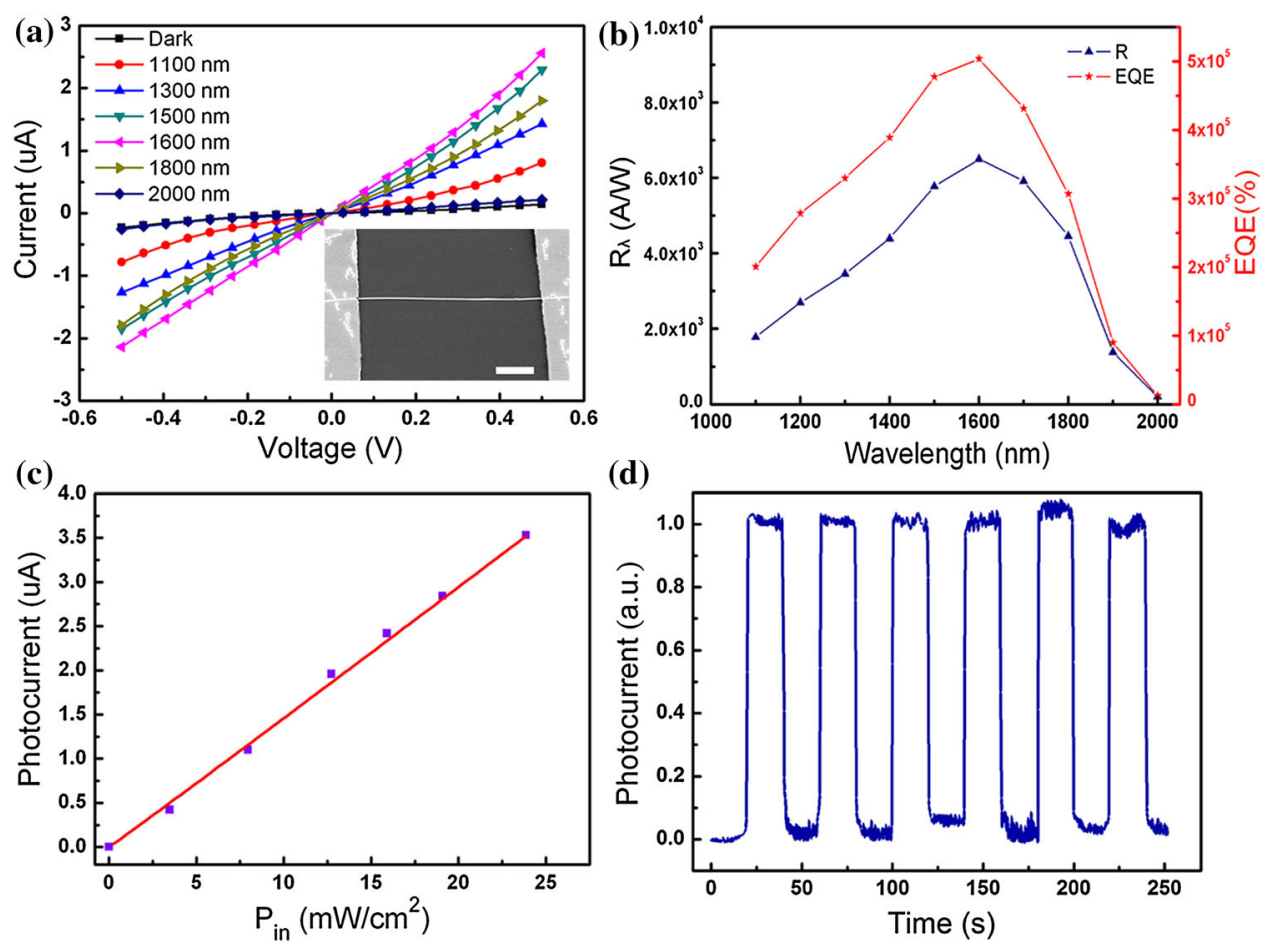

Fig. 5 a $I-V$ curves for a representative $\operatorname{In}_{0.65} \mathrm{Ga}_{0.35}$ As nanowire photodetector under light illumination with different wavelengths $($ Pin $=15.8$ $\mathrm{mW} \mathrm{cm}{ }^{-2}$ ) at room temperature, inset, SEM image of a fabricate photodetector, the scale bar is $2 \mu \mathrm{m}$. b The corresponding R and EQE versus incident wavelength of the photodetectors. $\mathbf{c}$ The photocurrent dependence of the incident light power density at a bias voltage of $0.5 \mathrm{~V}$. $\mathbf{d}$ Timedependent photocurrent response to $1600 \mathrm{~nm}$ light under $0.5 \mathrm{~V}$ bias

scattering and carrier trapping will reduce. This will result in a less $T_{\mathrm{r}}[28]$. The nanowires in higher quality of defect-free single crystal can facilitate the transport of the carrier along the axis of the wires and therefore increase the photocurrent gain [29]. It can be concluded that the electron trapping at the nanowire surface and one-dimensional structure with higher quality are two key elements to affect the carrier drift for a photocurrent gain. In addition, the electrode distance and Schottky contact may also affect the photoresponse. The shorter distance of $10 \mu \mathrm{m}$ between the two electrodes benefits to shorten the carrier transit time during the transport process. Moreover, the Schottky barriers in metal-semiconductor-metal (MSM) will reduce due to the photo-generated carriers and also benefits the electron injection [15, 30]. All of these elements can increase the photocurrent density effectively, and therefore lead to an enhancement of photoresponse.

Figure $5 \mathrm{c}$ displays the dependence of photocurrent on light intensity curves measured at a voltage of $0.5 \mathrm{~V}$. It demonstrates a good linear relationship between the photocurrent and the light intensity, which is beneficial for application in light power detection. This indicates that the $\mathrm{In}_{0.65} \mathrm{Ga}_{0.35} \mathrm{As}$ nanowire is a typical photon-dependent resistor. There may exist complex processes of carrier generation, trapping, and recombination in the nanowires [31]. The higher photoresponse is mainly due to its inner photoelectrical effect [9]. Figure 5d shows the time-related reproducible response of the photocurrent to illumination at $1600 \mathrm{~nm}$. One can see that the photodetector is stable under an optical power of $15.8 \mathrm{~mW} \mathrm{~cm} \mathrm{~cm}^{-2}$ and a bias voltage of $0.5 \mathrm{~V}$. The response time and recovery time, defined as the time between 10 and $90 \%$ of maximum photocurrent, is respective 70 and $280 \mathrm{~ms}$ (the detailed curves of photocurrent changing with time see Fig. S2). These values are comparable with those of InAs nanowire photodetectors [7].

\section{Conclusion}

In summary, single-crystal $\mathrm{In}_{0.65} \mathrm{Ga}_{0.35}$ As nanowires were synthesized by a simple CVD method. The nanowires have strong light emission in the near-infrared region. Photodetector based on as-grown nanowires was constructed and it exhibits a good photoresponse over a broad range from 1100 to $2000 \mathrm{~nm}$. A higher responsivity of $6.5 \times 10^{3}$ $\mathrm{A} / \mathrm{W}$ and external quantum efficiency of $5.04 \times 10^{5} \%$ were observed. The photodetector in this work may have 
potential applications in integrated optoelectronic devices for infrared radiation imaging, sensing, and information communications.

Acknowledgments The authors are grateful to the NSF of China (Nos. 61574054, 61505051, 11374092, 11204073, 61474040, and 51302077), the National Basic Research Program of China (No. 2012CB932703), the Hunan province science and technology plan (No. 2014FJ2001, 2014GK3015, and 2014TT1004), the Hunan Provincial Natural Science Foundation of China (No. 2015JJ3049), and the Aid program for Science and Technology Innovative Research Team in Higher Educational Institutions of Hunan Province.

Open Access This article is distributed under the terms of the Creative Commons Attribution 4.0 International License (http://crea tivecommons.org/licenses/by/4.0/), which permits unrestricted use, distribution, and reproduction in any medium, provided you give appropriate credit to the original author(s) and the source, provide a link to the Creative Commons license, and indicate if changes were made.

\section{References}

1. X.F. Duan, Y. Huang, R. Agarwal, C.M. Lieber, Single-nanowire electrically driven lasers. Nature 421(6920), 241-245 (2003). doi:10.1038/nature01353

2. A.L. Pan, W. Zhou, E.S.P. Leong, R. Liu, A.H. Chin, B.S. Zou, C.Z. Ning, Continuous alloy-composition spatial grading and superbroad wavelength-tunable nanowire lasers on a single chip. Nano Lett. 9(2), 784-788 (2009). doi:10.1021/n1803456k

3. J.Y. Xu, L. Ma, P.F. Guo, X.J. Zhuang, X.L. Zhu, W. Hu, X.F. Duan, A.L. Pan, Room-temperature dual-wavelength lasing from single nanoribbon lateral heterostructures. J. Am. Chem. Soc. 134(30), 12394-12397 (2012). doi:10.1021/ja3050458

4. T. Katsuhiro, Y. Masatoshi, F. Takashi, A III-V nanowire channel on silicon for high-performance vertical transistors. Nature 488(7410), 189-192 (2012). doi:10.1038/nature11293

5. Y.Y. Cao, Z.M. Wu, J.C. Ni, W.A. Bhutto, J. Li, S.P. Li, K. Huang, J.Y. Kang, Type-II core/shell nanowire heterostructures and their photovoltaic applications. Nano-Micro Lett. 4(3), 135-141 (2012). doi:10.3786/nml.v4i3.p135-141

6. M.X. Wang, G.H. Yue, Y.D. Lin, X. Wen, D.L. Peng, Z.R. Geng, Synthesis, optical properties and photovoltaic application of the $\mathrm{SnS}$ quasi-one-dimensional nanostructures. Nano-Micro Lett. 5(1), 1-6 (2013). doi:10.3786/nml.v5i1.p1-6

7. N. Guo, W.D. Hu, L. Liao, S. Yip, J.C. Ho et al., Anomalous and highly efficient InAs nanowire phototransistors based on majority carrier transport at room temperature. Adv. Mater. 26(48), 8203-8209 (2014). doi:10.1002/adma.201403664

8. P. Ren, W. Hu, Q. Zhang, X. Zhu, X. Zhuang et al., Band-selective infrared photodetectors with complete-composition-range $\operatorname{InAs}_{\mathrm{x}} \mathrm{P}_{1-\mathrm{x}}$ alloy nanowires. Adv. Mater. 26(44), 7444-7499 (2014). doi:10.1002/adma.201402945

9. Q.L. Li, Y. Li, J. Gao, S.D. Wang, X.H. Sun, High performance single $\operatorname{In}_{2} \mathrm{Se}_{3}$ nanowire Photodetector. Appl. Phys. Lett. 99(24), 243105 (2011). doi:10.1063/1.3669513

10. L. Ma, W. Hu, Q. Zhang, P. Ren, X. Zhuang et al., Room-temperature near-infrared photodetectors based on single heterojunction nanowires. Nano Lett. 14(2), 694-698 (2014). doi:10. 1021/nl403951f

11. K.M. Deng, L. Li, CdS nanoscale photodetectors. Adv. Mater. 26(17), 2619-2635 (2014). doi:10.1002/adma.201304621
12. X.Q. Liu, X. Liu, J.L. Wang, C.N. Liao, X.H. Xiao et al., Scalable integration of indium zinc oxide/photosensitive-nanowire composite thin-film transistors for transparent multicolor photodetectors array. Adv. Mater. 26(18), 2919-2924 (2014). doi:10. 1002/adma.201305073

13. X.M. Xie, G.Z. Shen, Single-crystalline $\operatorname{In}_{2} \mathrm{~S}_{3}$ nanowire-based flexible visible-light photodetectors with an ultra-high photoresponse. Nanoscale 7(11), 5046-5052 (2015). doi:10.1039/ C5NR00410A

14. R. Basori, A.K. Raychaudhuri, Role of contact and contact modification on photo-response in a charge transfer complex single nanowire device. Nano-Micro Lett. 6(1), 63-69 (2014). doi:10.5101/nml.v6i1.p63-69

15. C.-H. Kuo, J.-M. Wu, S.-J. Lin, W.-C. Chang, High sensitivity of middle-wavelength infrared photodetectors based on an individual InSb nanowire. Nanoscale Res. Lett. 8(1), 327 (2013). doi:10. 1186/1556-276X-8-327

16. J.S. Miao, W.D. Hu, N. Guo, Z.Y. Lu, X.M. Zou et al., Single InAs nanowire room-temperature near-infrared photodetectors. ACS Nano 8(4), 3628-3635 (2014). doi:10.1021/nn500201g

17. Z. Liu, T. Luo, B. Liang, G. Chen, G. Yu, X.M. Xie, D. Chen, G.Z. Shen, High-detectivity InAs nanowire photodetectors with spectral response from ultraviolet to near-infrared. Nano Res. 6(11), 775-783 (2013). doi:10.1007/s12274-013-0356-0

18. Y. Hou, J.R. Liu, M. Buchanan, A.J.S. Thorpe, P.J. Poole, H.C. Liu, K. Wu, S. Roorda, X.P. Zhang, Terahertz generation using implanted InGaAs photomixers and multi-wavelength quantum dot lasers. Nano-Micro Lett. 4(1), 10-13 (2012). doi:10.3786/ nml.v4i1.p10-13

19. J.C. Shin, K.H. Kim, K.J. Yu, H.F. Hu, L.J. Yin, C.Z. Ning, J.A. Rogers, J.-M. Zuo, X.L. Li, $\operatorname{In}_{x} \mathrm{Ga}_{1-\mathrm{x}}$ As nanowires on silicon: one-dimensional heterogeneous epitaxy, bandgap engineering, and photovoltaics. Nano Lett. 11(11), 4831-4838 (2011). doi:10. $1021 / \mathrm{nl} 202676 \mathrm{~b}$

20. J.J. Hou, F.Y. Wang, N. Han, F. Xiu, S.P. Yip, M. Fang, H. Lin, F.T. Hung, J.C. Ho, Stoichiometric effect on electrical, optical, and structural properties of composition-tunable $\mathrm{In}_{\mathrm{x}} \mathrm{Ga}_{1-\mathrm{x}} \mathrm{As}$ nanowires. ACS Nano 6(10), 9320-9325 (2012). doi:10.1021/ nn304174g

21. K.A. Dick, K. Deppert, L.S. Karlsson, L.R. Wallenberg, L. Samuelson, W. Seifert, A new understanding of Au-assisted growth of III-V semiconductor nanowires. Adv. Funct. Mater. 15(10), 1603-1610 (2005). doi:10.1002/adfm.200500157

22. J. Groenen, R. Carles, G. Landa, Optical-phonon behavior in $\mathrm{Ga}_{1-\mathrm{x}} \mathrm{In}_{\mathrm{x}}$ As: the role of microscopic strains and ionic plasmon coupling. Phys. Rev. B 58(16), 10452-10462 (1998). doi:10. 1103/PhysRevB.58.10452

23. C.S. Jung, H.S. Kim, G.B. Jung, K.J. Gong, Y.J. Cho, S.Y. Jang, C.H. Kim, C.-W. Lee, J. Park, Composition and phase tuned InGaAs alloy nanowires. J. Phys. Chem. C 115(16), 7843-7850 (2011). doi:10.1021/jp2003276

24. S. Morkötter, S. Funk, M. Liang, M. Döblinger, S. Hertenberger et al., Role of microstructure on optical properties in high-uniformity $\mathrm{In}_{1-\mathrm{x}} \mathrm{Ga}_{\mathrm{x}}$ As nanowire arrays: evidence of a wider wurtzite band gap. Phys. Rev. B 87(20), 205303 (2013). doi:10.1103/ PhysRevB.87.205303

25. Y.P. Varshni, Temperature dependence of the energy gap in semiconductors. Physica 34(1), 149-154 (1967). doi:10.1016/ 0031-8914(67)90062-6

26. T. Umezawa, K. Akahane, N. Yamamoto, A. Kanno, T. Kawanishi, Highly sensitive photodetector using ultra-high-density $1.5-\mu \mathrm{m}$ quantum dots for advanced optical fiber communications. IEEE 20(6), 3801907 (2014). doi:10.1109/JSTQE.2014. 2321288

27. Y.L. Lee, C.C. Huang, C.L. Ho, M.C. Wu, Planar InGaAs p-i-n photodiodes with transparent-conducting-based antireflection and 
double-path reflector. IEEE Electron Device Lett. 34(11), 1406-1408 (2013). doi:10.1109/LED.2013.2281830

28. Z. Liu, G. Chen, B. Liang, G. Yu, H.T. Huang, D. Chen, G.Z. Shen, Fabrication of high-quality ZnTe nanowires toward highperformance rigid/flexible visible-light photodetectors. Opt. Express 21(6), 7799-7810 (2013). doi:10.1364/OE.21.007799

29. C. Soci, A. Zhang, X.-Y. Bao, H. Kim, Y. Lo, D.L. Wang, Nanowire photodetectors. J. Nanosci. Nanotechnol. 10(3), 1430-1499 (2010). doi:10.1166/jnn.2010.2157
30. R. Basori, K. Das, P. Kumar, K.S. Narayan, A.K. Raychaudhuri, Single CuTCNQ charge transfer complex nanowire as ultra high responsivity photodetector. Opt. Express 22(5), 4944-4952 (2014). doi:10.1364/OE.22.004944

31. H. Kind, H.Q. Yan, B. Messer, M. Law, P.D. Yang, Nanowire ultraviolet photodetectors and optical switches. Adv. Mater. 14(2), 158-160 (2002). doi:10.1002/1521-4095(20020116)14: $2<158$ :AID-ADMA158>3.0.CO;2-W 